\title{
A Plethysm formula on the characteristic map of induced linear characters from $U_{n}\left(\mathbb{F}_{q}\right)$ to $G L_{n}\left(\mathbb{F}_{q}\right)$
}

\author{
Zhi Chen \\ Department of Mathematics and Statistics \\ York University \\ Toronto, ON, Canada \\ czhi@mathstat .yorku.ca
}

Submitted: May 19, 2012; Accepted: Aug 2, 2013; Published: Aug 9, 2013

Mathematics Subject Classifications: 05E10, 20C33

\begin{abstract}
This paper gives a plethysm formula on the characteristic map of the induced linear characters from the unipotent upper-triangular matrices $U_{n}\left(\mathbb{F}_{q}\right)$ to $G L_{n}\left(\mathbb{F}_{q}\right)$, the general linear group over finite field $\mathbb{F}_{q}$. The result turns out to be a multiple of a twisted version of the Hall-Littlewood symmetric functions $\tilde{P}_{n}[Y ; q]$. A recurrence relation is also given which makes it easy to carry out the computation.
\end{abstract}

Keywords: representation theory; induced characters; symmetric functions; supercharacter theory

\section{Introduction}

Let $\mathbb{F}_{q}$ be a fixed finite field and $G L_{n}\left(\mathbb{F}_{q}\right)$ the finite general linear group over $\mathbb{F}_{q}$. The representation theory of $G L_{n}\left(\mathbb{F}_{q}\right)$ over $\mathbb{C}$ was presented by J.A.Green [5]. He also constructed the characteristic map which builds a connection between the character spaces of $G L_{n}\left(\mathbb{F}_{q}\right)$ for $n \geqslant 0$ and the Cartesian product over infinitely indexed sets of rings of symmetric functions. In character theory, the study of induced linear characters from subgroups is very useful in order to understand the character ring of the larger group.

In this paper, we consider certain induced linear characters from the group of unipotent upper-triangular matrices $U_{n}\left(\mathbb{F}_{q}\right)$ to $G L_{n}\left(\mathbb{F}_{q}\right)$. The representations of these induced linear characters are known as Gelfand-Graev modules, which play an important role in the representation theory of finite groups of Lie type ([4], [12]). The formula for the characteristic map of the induced linear characters is given by Thiem [9]. We then apply 
a homomorphism $\rho$ (see Definition 5) on the image of the characteristic map. We refer to the result as a plethysm formula because it involves a composition of symmetric functions using the plethysm operation. There are two advantages in doing so: to get a simpler formula and to express the result as a multiple of a twisted version of the Hall-Littlewood symmetric functions $\tilde{P}_{n}[Y ; q]$. We hope this method could contribute to the study of the irreducible decomposition of the induced characters from $U_{n}\left(\mathbb{F}_{q}\right)$ to $G L_{n}\left(\mathbb{F}_{q}\right)$.

In section 2 we give some background knowledge on symmetric functions and representation theory of $G L_{n}\left(\mathbb{F}_{q}\right)$ and $U_{n}\left(\mathbb{F}_{q}\right)$. Since the character theory of $U_{n}\left(\mathbb{F}_{q}\right)$ is known as a wild problem, supercharacter theory is built up as an approximation of the ordinary character theory. The linear characters of $U_{n}\left(\mathbb{F}_{q}\right)$ that we are considering are part of the category of supercharcters of $U_{n}\left(\mathbb{F}_{q}\right)$. We introduce further questions about the induction of all supercharacters in Section 4. In Section 3 we give our main result about the plethysm formula. A natural recurrence relation is obtained so that we can carry out the computation of the homomorphism $\rho$ on the characteristic map of the induced linear characters more easily. We also give a relation between the characteristic map of the induced characters from $U_{n}\left(\mathbb{F}_{q}\right)$ to $G L_{n}\left(\mathbb{F}_{q}\right)$, and the homomorphism $\rho$ on those characteristics. This is depicted in the following diagram

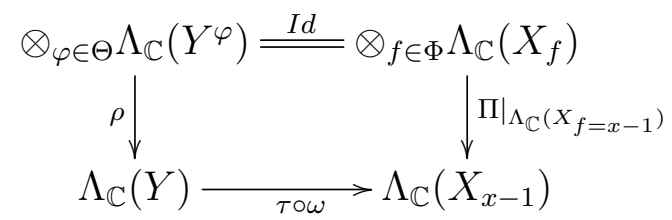

where the notation is explained in Theorem 17. From the above commutative diagram we show that our simplified plethysm formula does not lose any information on the characteristic map of the induced characters from $U_{n}\left(\mathbb{F}_{q}\right)$ to $G L_{n}\left(\mathbb{F}_{q}\right)$.

\section{Background}

\subsection{Symmetric functions}

The notation in this paper follows closely the book of Macdonald [7] and Thiem [10].

Definition 1. A partition $\lambda$ of $n \in \mathbf{N}$, is a sequence $\lambda=\left(\lambda_{1}, \lambda_{2}, \ldots, \lambda_{l}\right)$ of positive integers in weakly decreasing order: $\lambda_{1} \geqslant \lambda_{2} \geqslant \cdots \geqslant \lambda_{l}$, such that $\lambda_{1}+\lambda_{2}+\cdots+\lambda_{l}=n$. We denote this by $\lambda \vdash n$. Here, each $\lambda_{i}(1 \leqslant i \leqslant l)$ is called a part of $\lambda$. We say the length of the partition $\lambda$ is $l=l(\lambda)$, which is the number of parts of $\lambda$. We use $|\lambda|$ to denote the sum of all parts $\lambda_{1}+\lambda_{2}+\cdots+\lambda_{l}$, and we call $|\lambda|$ the size of the partition. Sometimes we also use the notation:

$$
\lambda=\left(1^{m_{1}}, 2^{m_{2}}, \ldots, n^{m_{n}}, \ldots\right),
$$

where each $m_{i}$ means there are $m_{i}$ parts in $\lambda$ equal to $i$.

Let $\Lambda_{\mathbb{C}}(Y)$ denote the ring of symmetric functions with complex coefficients in the variables $Y=\left\{y_{1}, y_{2}, \ldots\right\}$. We denote the complete symmetric functions, elementary 
symmetric functions, monomial symmetric functions, power-sum symmetric functions, and Schur symmetric functions by $h_{\lambda}[Y], e_{\lambda}[Y], m_{\lambda}[Y], p_{\lambda}[Y]$, and $s_{\lambda}[Y]$ respectively.

Following other references (e.g. Garsia and Tesler [3]), we use the notation $\Omega$ for the basic symmetric function kernel. Then the generating function for $h_{n}[Y]$ is

$$
\Omega[t Y]=\sum_{n \geqslant 0} h_{n}[Y] t^{n}=\prod_{j \geqslant 1}\left(1-y_{j} t\right)^{-1}
$$

It is also well known that

$$
\Omega[X] \Omega[Y]=\Omega[X+Y] \text {, and } \Omega[\mathrm{X}] / \Omega[\mathrm{Y}]=\Omega[\mathrm{X}-\mathrm{Y}]
$$

Let $X=\left\{x_{1}, x_{2}, \ldots\right\}$ be another set of finite or infinite variables. We have the following identity:

$$
\Omega[X Y]:=\prod_{i, j}\left(1-x_{i} y_{j}\right)^{-1}=\sum_{\lambda} m_{\lambda}[X] h_{\lambda}[Y]
$$

summed over all partitions $\lambda$.

There is a scalar product defined on $\Lambda_{\mathbb{C}}(Y)$, which makes $\left(m_{\lambda}\right)$ and $\left(h_{\lambda}\right)$ dual to each other:

$$
\left\langle h_{\lambda}, m_{\mu}\right\rangle=\delta_{\lambda \mu}
$$

for all partitions $\lambda, \mu$, where $\delta_{\lambda \mu}$ is the Kronecker delta.

We use $P_{\lambda}[Y ; t]$ to denote the Hall-Littlewood symmetric functions, as defined in [7] (see page 209). If we define

$$
\begin{aligned}
\boldsymbol{q}_{r} & =\boldsymbol{q}_{r}[Y ; t]=(1-t) P_{(r)}[Y ; t] \text { for } r \geqslant 1, \\
\boldsymbol{q}_{0} & =\boldsymbol{q}_{0}[Y ; t]=1,
\end{aligned}
$$

then the generating function for $\boldsymbol{q}_{r}[Y ; t]$ is

$$
Q(u)=\sum_{r \geqslant 0} \boldsymbol{q}_{r}[Y ; t] u^{r}=\prod_{i} \frac{1-y_{i} t u}{1-y_{i} u}=\Omega[(1-t) u Y] .
$$

For each partition $\lambda$, let $n(\lambda)=\sum_{i \geqslant 1}(i-1) \lambda_{i}$. Define

$$
\tilde{P}_{\lambda}[Y ; q]=q^{-n(\lambda)} P_{\lambda}\left[Y ; q^{-1}\right]
$$

and we call $\tilde{P}_{\lambda}[Y ; q]$ the twisted Hall-Littlewood symmetric functions.

From [7], it is well known that the plethysm can be defined by

$$
h_{a}\left[p_{b}[Y]\right]=h_{a}\left[y_{1}^{b}, y_{2}^{b}, \ldots\right]
$$

which is the coefficient of $t^{a b}$ in $\prod_{j \geqslant 1}\left(1-y_{j}^{b} t^{b}\right)^{-1}$. 


\subsection{Representation theory of $G L_{n}\left(\mathbb{F}_{q}\right)$}

The representation theory of the finite general linear group $G_{n}=G L_{n}\left(\mathbb{F}_{q}\right)$ over $\mathbb{C}$ can be found in J.A.Green [5], Macdonald [7] and Thiem [9]. Here we give a short description of the characteristic map constructed by J.A.Green.

Let $\overline{\mathbb{F}}_{q}$ denote the algebraic closure of the finite field $\mathbb{F}_{q}$. The multiplicative group of $\overline{\mathbb{F}}_{q}$ is denoted by $\overline{\mathbb{F}}_{q}^{\times}$. The Frobenius automorphism of $\overline{\mathbb{F}}_{q}$ over $\mathbb{F}_{q}$ is given by

$$
F: x \rightarrow x^{q} \text {, where } x \in \overline{\mathbb{F}}_{q} .
$$

For each $n \geqslant 1$, we use $\overline{\mathbb{F}}_{q, n}^{\times}$to denote the fixed points of $F^{n}$ in $\overline{\mathbb{F}}_{q}^{\times}$.

Let $\overline{\mathbb{F}}_{q}^{*}=\left\{\phi: \overline{\mathbb{F}}_{q}^{\times} \rightarrow \mathbb{C}^{\times}\right\}$be the group of complex-valued multiplicative characters of $\overline{\mathbb{F}}_{q}^{\times}$. The Frobenius automorphism on $\overline{\mathbb{F}}_{q}^{*}$ is

$$
F: \xi \rightarrow \xi^{q} \text {, where } \xi \in \overline{\mathbb{F}}_{q}^{*}
$$

For each $n \geqslant 1$, let $\overline{\mathbb{F}}_{q, n}^{*}$ be the group of elements fixed by $F^{n}$. We also define a pairing of $\overline{\mathbb{F}}_{q}^{*}$ with $\overline{\mathbb{F}}_{q}^{\times}$by

$$
\langle\xi, x\rangle_{n}=\xi(x)
$$

for $\xi \in \overline{\mathbb{F}}_{q, n}^{*}$ and $x \in \overline{\mathbb{F}}_{q, n}^{\times}$.

We then define

$$
\Phi=\left\{F \text {-orbits of } \overline{\mathbb{F}}_{q}^{\times}\right\} \text {and } \Theta=\left\{F \text {-orbits of } \overline{\mathbb{F}}_{q}^{*}\right\} .
$$

Since each $F$-orbit of $\overline{\mathbb{F}}_{q}^{\times}$is in one-to-one correspondence with an irreducible polynomial over $\mathbb{F}_{q}$, we can also use $f$ to denote each $F$-orbit in $\Phi$. A partition-valued function $\boldsymbol{\mu}$ on $\Phi$ is a function which maps each $f \in \Phi$ to a partition $\boldsymbol{\mu}(f)$. The size of $\boldsymbol{\mu}$ is

$$
\|\boldsymbol{\mu}\|=\sum_{f \in \Phi} d(f)|\boldsymbol{\mu}(f)|
$$

where $d(f)$ is equal to the degree of $f \in \Phi$.

Let $\mathbb{P}$ denote the set of all partitions and

$$
\mathcal{P}^{\Phi}=\bigcup_{n \geqslant 0} \mathcal{P}_{n}^{\Phi}, \text { where } \mathcal{P}_{n}^{\Phi}=\{\boldsymbol{\mu}: \Phi \rightarrow \mathbb{P} ;\|\boldsymbol{\mu}\|=n\}
$$

We use $K^{\boldsymbol{\mu}}$ to denote the conjugacy classes in $G_{n}$ parameterized by $\boldsymbol{\mu} \in \mathcal{P}_{n}^{\Phi}[7]$. The characteristic function of the conjugacy class $K^{\mu}$ is denoted by $\pi_{\boldsymbol{\mu}}$.

Similarly, for each partition-valued function $\boldsymbol{\lambda}: \Theta \rightarrow \mathbb{P}$, the size of $\boldsymbol{\lambda}$ is

$$
\|\boldsymbol{\lambda}\|=\sum_{\varphi \in \Theta} d(\varphi)|\boldsymbol{\lambda}(\varphi)|
$$

where $d(\varphi)$ is equal to the number of elements in $\varphi$. Let

$$
\mathcal{P}^{\Theta}=\bigcup_{n \geqslant 0} \mathcal{P}_{n}^{\Theta}, \text { where } \mathcal{P}_{n}^{\Theta}=\{\boldsymbol{\lambda}: \Theta \rightarrow \mathbb{P} ;\|\boldsymbol{\lambda}\|=n\} .
$$


We use $G_{n}^{\boldsymbol{\lambda}}$ to denote the irreducible $G_{n}$-modules indexed by $\boldsymbol{\lambda} \in \mathcal{P}_{n}^{\Theta}[7]$. The character of the irreducible $G_{n}$-module $G_{n}^{\boldsymbol{\lambda}}$ is denoted by $\chi^{\boldsymbol{\lambda}}$.

For every $f \in \Phi$, let $X_{f}:=\left\{X_{1, f}, X_{2, f}, \ldots\right\}$ be a set of infinitely many variables. Each $X_{i, f}$ has degree $d(f)$.

Let

$$
\tilde{P}_{\eta}[f]=\tilde{P}_{\eta}\left[X_{f} ; q^{d(f)}\right]=q^{-d(f) n(\eta)} P_{\eta}\left[X_{f} ; q^{-d(f)}\right],
$$

where $\tilde{P}_{\eta}\left[X_{f} ; q^{d(f)}\right]$ is the twisted Hall-Littlewood symmetric function. Define

$$
\tilde{P}_{\boldsymbol{\mu}}=\prod_{f \in \Phi} \tilde{P}_{\boldsymbol{\mu}(f)}[f]
$$

For every $\varphi \in \Theta$, let $Y^{\varphi}:=\left\{Y_{1}^{\varphi}, Y_{2}^{\varphi}, \ldots\right\}$ be a set of infinitely many variables. Each $Y_{i}^{\varphi}$ has degree $d(\varphi)$. Define

$$
S_{\boldsymbol{\lambda}}=\prod_{\varphi \in \Theta} s_{\boldsymbol{\lambda}(\varphi)}\left[Y^{\varphi}\right]
$$

where $s_{\boldsymbol{\lambda}(\varphi)}\left[Y^{\varphi}\right]$ is the Schur symmetric function.

We define the transformation between the symmetric functions in the variables $\left\{X_{f}\right.$ : $f \in \Phi\}$ and those in the variables $\left\{Y^{\varphi}: \varphi \in \Theta\right\}$ by the following identity:

$$
p_{k}\left[Y^{\varphi}\right]=(-1)^{n-1} \sum_{x \in \overline{\mathbb{F}}_{q, n}^{\times}} \xi(x) p_{n / d\left(f_{x}\right)}\left[X_{f_{x}}\right] \text {, }
$$

where $n=k \times d(\varphi), \xi$ is any element in $\varphi$, and $f_{x}$ is the irreducible polynomial that contains $x$ as a root. Since $f_{x}=f_{y}$ if $x, y$ are in the same $F$-orbit in $\overline{\mathbb{F}}_{q, n}^{\times}$, equation (2.4) is well defined for any $\xi \in \varphi$. Also here $p_{k}\left[Y^{\varphi}\right]$ and $p_{n / d\left(f_{x}\right)}\left[X_{f_{x}}\right]$ are power sums in different sets of variables. Equation (2.4) is an isomorphism and its inverse can be found in [7]. For interested readers willing to know more details, please refer to [7] and [11].

Since the power-sum symmetric functions form a basis of the ring of symmetric functions, with the basis transformation given by equation (2.4), we let

$$
\Lambda_{\mathbb{C}}=\otimes_{f \in \Phi} \Lambda_{\mathbb{C}}\left(X_{f}\right)=\otimes_{\varphi \in \Theta} \Lambda_{\mathbb{C}}\left(Y^{\varphi}\right),
$$

where $\Lambda_{\mathbb{C}}\left(X_{f}\right)$ is the ring of symmetric functions in $X_{f}$, and $\Lambda_{\mathbb{C}}\left(Y^{\varphi}\right)$ is the ring of symmetric functions in $Y^{\varphi}$. As a graded ring, we have

$$
\begin{aligned}
\Lambda_{\mathbb{C}} & =\mathbb{C}-\operatorname{span}\left\{\tilde{P}_{\boldsymbol{\mu}} \mid \boldsymbol{\mu} \in \mathcal{P}^{\Phi}\right\} \\
& =\mathbb{C}-\operatorname{span}\left\{S_{\boldsymbol{\lambda}} \mid \boldsymbol{\lambda} \in \mathcal{P}^{\Theta}\right\} .
\end{aligned}
$$

From [7] we know that the conjugacy classes $K^{\boldsymbol{\mu}}$ of $G_{n}$ are parameterized by $\boldsymbol{\mu} \in \mathcal{P}_{n}^{\Phi}$, and the irreducible characters $\chi^{\boldsymbol{\lambda}}$ of $G_{n}$ are indexed by $\boldsymbol{\lambda} \in \mathcal{P}_{n}^{\Theta}$. The following theorem gives the characteristic map of $G_{n}$. 
Theorem 2. (Green [5], Macdonald [7], Zelevinski [14]) Let $A_{n}$ denote the space of complex-valued class functions on $G_{n}$ and $A=\oplus_{n \geqslant 0} A_{n}$. The linear map

$$
\begin{aligned}
c h: A & \longrightarrow \Lambda_{\mathbb{C}} \\
\chi^{\boldsymbol{\lambda}} & \mapsto S_{\boldsymbol{\lambda}}, \quad \text { for } \boldsymbol{\lambda} \in \mathcal{P}^{\Theta}, \\
\pi_{\boldsymbol{\mu}} & \mapsto \tilde{P}_{\boldsymbol{\mu}}, \quad \text { for } \boldsymbol{\mu} \in \mathcal{P}^{\Phi},
\end{aligned}
$$

is a Hopf algebra isomorphism.

\subsection{Supercharacter theory}

Let $U_{n}$ be the group of unipotent upper-triangular matrices with entries in the finite field $\mathbb{F}_{q}$, and ones on the diagonal. This group is the subgroup of the finite general linear group $G_{n}$. Although the character theory on $U_{n}$ is a wild problem, people came up with a slightly coarser version called superclass and supercharacter theory (André [2], Yan [13]). This approximation of the character theory of $U_{n}$ is relatively easier to study and compute. Superclasses are certain unions of conjugacy classes and supercharacters are sums of irreducible characters. They are compatible in the sense that supercharacters are constant on superclasses. The supercharacter theory has a rich combinatorial structure (ref. [10]) and connects to some other algebraic structures as well (ref. [1]).

The superclasses of $U_{n}$ can be indexed by the $\mathbb{F}_{q}^{\times}$-labeled set partitions, and a supercharacter becomes an irreducible character if the corresponding indexed $\mathbb{F}_{q}^{\times}$-labeled set partition has no crossing arcs. For the strict definitions and more details on supercharacters please see [10] or [1].

In this paper we consider the linear supercharacters of $U_{n}$ indexed by

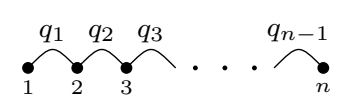

where $q_{1}, \ldots, q_{n-1} \in \mathbb{F}_{q}^{\times}$(see Thiem [10] for this notation). Let $\chi_{\left(q_{1}, \ldots, q_{n-1}\right)}^{(n)}$ denote the above character. We induce $\chi_{\left(q_{1}, \ldots, q_{n-1}\right)}^{(n)}$ from $U_{n}$ to $G_{n}$ by the formula

$$
\chi_{\left(q_{1}, \ldots, q_{n-1}\right)}^{(n)} \uparrow_{U_{n}}^{G_{n}}(g)=\frac{1}{\left|U_{n}\right|} \sum_{h \in G_{n}} \bar{\chi}_{\left(q_{1}, \ldots, q_{n-1}\right)}^{(n)}\left(h g h^{-1}\right)
$$

where $\bar{\chi}(s)=\chi(s)$ if $s \in U_{n}$, and $\bar{\chi}(s)=0$ if $s \notin U_{n}$.

The induced character $\chi_{\left(q_{1}, \ldots, q_{n-1}\right)}^{(n)} \uparrow_{U_{n}}^{G_{n}}$ is a character of $G_{n}$, which is known as the character of the Gelfand-Greav module ([4], [9]). Applying the homomorphism $\rho$ defined in Section 3 to the characteristic map of $\chi_{\left(q_{1}, \ldots, q_{n-1}\right)}^{(n)} \uparrow_{U_{n}}^{G_{n}}$, we get a multiple of the twisted Hall-Littlewood symmetric function $\tilde{P}_{n}$. An explicit formula for this result together with a recurrence relation is also given in Section 3. 


\section{Plethysm Formula for the induced character}

We start from the formula of the characteristic map of $\chi_{\left(q_{1}, \ldots, q_{n-1}\right)}^{(n)} \uparrow_{U_{n}}^{G_{n}}$, which is given by Thiem [9].

Theorem 3. (Thiem [9])

$$
\operatorname{ch}\left(\chi_{\left(q_{1}, \ldots, q_{n-1}\right)}^{(n)} \uparrow_{U_{n}}^{G_{n}}\right)=\sum_{\substack{\boldsymbol{\lambda} \in \mathcal{P}_{n}^{\Theta} \\ h t(\boldsymbol{\lambda})=1}} S_{\boldsymbol{\lambda}}
$$

where $h t(\boldsymbol{\lambda})=\max \{l(\boldsymbol{\lambda}(\varphi)) \mid \varphi \in \Theta\}$.

Notice that $h t(\boldsymbol{\lambda})=1$ implies for every $\varphi \in \Theta$ we have $l(\boldsymbol{\lambda}(\varphi)) \leqslant 1$, which means $\boldsymbol{\lambda}(\varphi)$ contains at most one part. Since for each $a \in \mathbb{N}, s_{a}\left[Y^{\varphi}\right]=h_{a}\left[Y^{\varphi}\right]$. From the definition of $S_{\boldsymbol{\lambda}}$, we can write $(3.1)$ as

$$
\begin{aligned}
\operatorname{ch}\left(\chi_{\left(q_{1}, \ldots, q_{n-1}\right)}^{(n)} \uparrow_{U_{n}}^{G_{n}}\right) & =\sum_{\substack{\boldsymbol{\lambda} \in \mathcal{P}^{\Theta} \\
h t(\boldsymbol{\lambda})=1}} \prod_{\substack{\theta \\
\varphi \in \Theta}} s_{\boldsymbol{\lambda}(\varphi)}\left[Y^{\varphi}\right]=\sum_{\substack{\boldsymbol{\lambda} \in \mathcal{P}^{\Theta} \\
h t(\boldsymbol{\lambda})=1}} \prod_{\substack{n \\
\varphi \in \Theta}} h_{\boldsymbol{\lambda}(\varphi)}\left[Y^{\varphi}\right] \\
& =\sum_{\substack{a_{1} b_{1}+\cdots+a_{k} b_{k}=n \\
\boldsymbol{\lambda}(\Theta)=\left\{a_{1}, \ldots, a_{k}\right\} \in \mathcal{P}_{n}^{\Theta}}} \sum_{\substack{d e g\left(\varphi_{i}\right)=b_{i} \\
\varphi_{1}, \ldots, \varphi_{k} \text { distinct }}} h_{a_{1}}\left[Y^{\varphi_{1}}\right] h_{a_{2}}\left[Y^{\varphi_{2}}\right] \cdots h_{a_{k}}\left[Y^{\varphi_{k}}\right],
\end{aligned}
$$

where $Y^{\varphi_{1}}, Y^{\varphi_{2}}, \ldots, Y^{\varphi_{k}}$ are different sets of variables. For $i$ from 1 to $k$, each variable in the set $Y^{\varphi_{i}}=\left\{Y_{1}^{\varphi_{i}}, Y_{2}^{\varphi_{i}}, \ldots\right\}$ has degree $b_{i}$.

We give an example to better understand formulae (3.1) and (3.2).

Example 4. For $n=3$, we have

$$
\begin{aligned}
\operatorname{ch}\left(\chi_{\left(q_{1}, q_{2}\right)}^{(3)} \uparrow_{U_{3}}^{G_{3}}\right) & =\sum_{\substack{\varphi_{1}, \varphi_{2}, \varphi_{3} \text { distinct } \\
\operatorname{deg}\left(\varphi_{i}\right)=1}} h_{1}\left[Y^{\varphi_{1}}\right] h_{1}\left[Y^{\varphi_{2}}\right] h_{1}\left[Y^{\varphi_{3}}\right] \\
& +\sum_{\substack{\psi_{1}, \psi_{2} \text { distinct } \\
\operatorname{deg}\left(\psi_{i}\right)=1}} h_{2}\left[Y^{\psi_{1}}\right] h_{1}\left[Y^{\psi_{2}}\right]+\sum_{\substack{\operatorname{deg}\left(\bar{\varphi}_{1}\right)=2 \\
\operatorname{deg}\left(\bar{\varphi}_{2}\right)=1}} h_{1}\left[Y^{\bar{\varphi}_{1}}\right] h_{1}\left[Y^{\bar{\varphi}_{2}}\right] \\
& +\sum_{\operatorname{deg}(\varphi)=1} h_{3}\left[Y^{\varphi}\right]+\sum_{\operatorname{deg}(\psi)=3} h_{1}\left[Y^{\psi}\right] .
\end{aligned}
$$

From the above example we see that the expansion on the right-hand side of (3.2) becomes more complicated as $n$ increases. This inspires us to come up with the idea of using a homomorphism, as we will see later in Definition 5, to simplify the computation.

For each term in equation (3.2), we have a two row array $\left(\begin{array}{cccc}b_{1} & b_{2} & \cdots & b_{k} \\ a_{1} & a_{2} & \cdots & a_{k}\end{array}\right)$ where $b_{i}=d\left(\varphi_{i}\right)$ and it satisfies the condition $\sum_{i=1}^{k} a_{i} b_{i}=n$. We arrange the pairs $\left(b_{i}, a_{i}\right)$ such that:

(1) $b_{1} \leqslant b_{2} \leqslant \ldots \leqslant b_{k}$ 
(2) $a_{j} \leqslant a_{j+1}$ if $b_{j}=b_{j+1}$ for $1 \leqslant j<k$.

Once the array is sorted, we can denote it as follows:

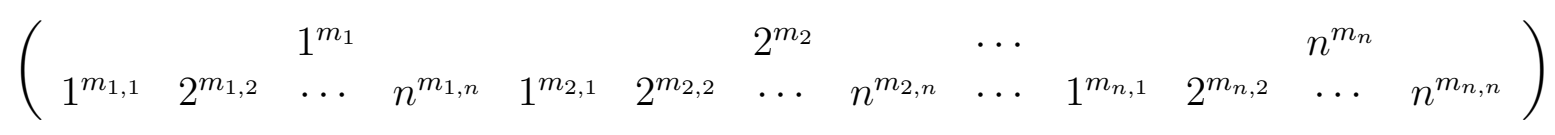

where $\sum_{i, j=1}^{n}\left(m_{i, j} \times j \times i\right)=n$ and $m_{i, 1}+m_{i, 2}+\ldots+m_{i, n}=m_{i}$ for $1 \leqslant i \leqslant n$. Each $m_{i}$ counts the number of different sets of variables appearing in the term with the same degree $i$. Each $m_{i, j}$ counts the number of complete symmetric functions $h_{j}$ in variables with degree $i$.

For a given $i$, let $l_{q}(i)$ denote the number of all different sets of variables with the same degree $i$. We know that $l_{q}(i)$ is equal to the number of irreducible polynomials $f$ over finite field $\mathbb{F}_{q}$ with degree $i$ and satisfying $f(0) \neq 0$. The number of irreducible polynomials of degree $i$ over $\mathbb{F}_{q}$ is given by the formula

$$
L_{q}(i)=\frac{1}{i} \sum_{d \mid i} \mu(d) q^{\frac{i}{d}}
$$

where $\mu$ is the Möbius function. Then we have

$$
l_{q}(i)= \begin{cases}L_{q}(1)-1, & \text { for } i=1 \\ L_{q}(i), & \text { for } i \geqslant 2 .\end{cases}
$$

Thus for a given $i$ and a list of numbers $\left[m_{i, 1}, m_{i, 2}, \ldots, m_{i, n}\right]$ where $m_{i, 1}+m_{i, 2}+\ldots+m_{i, n}=$ $m_{i}$, the number of products of the form

$$
\begin{aligned}
& h_{1}\left[Y^{\varphi_{i, 1}}\right] h_{1}\left[Y^{\varphi_{i, 2}}\right] \cdots h_{1}\left[Y^{\varphi_{i, m_{i, 1}}}\right] \\
& \times h_{2}\left[Y^{\varphi_{i, m_{i, 1}+1}}\right] h_{2}\left[Y^{\varphi_{i, m_{i, 1}+2}}\right] \cdots h_{2}\left[Y^{\varphi_{i, m_{i, 1}+m_{i, 2}}}\right] \\
& \times \cdots \\
& \times h_{n}\left[Y^{\varphi_{i, m_{i, 1}+\cdots+m_{i, n-1}+1}}\right] h_{n}\left[Y^{\varphi_{i, m_{i, 1}+\cdots+m_{i, n-1}+2}}\right] \cdots h_{n}\left[Y^{\varphi_{i, m_{i}}}\right]
\end{aligned}
$$

is equal to

$$
\frac{l_{q}(i)\left(l_{q}(i)-1\right) \cdots\left(l_{q}(i)-m_{i}+1\right)}{m_{i, 1} ! m_{i, 2} ! \cdots m_{i, n} !},
$$

where $Y^{\varphi_{i, 1}}, Y^{\varphi_{i, 2}}, \ldots, Y^{\varphi_{i, m_{i}}}$ are $m_{i}$ different sets of variables with the same degree $i$. Notice that when $n$ increases, we get more terms on the right-hand side of equation (3.2).

In order to simplify the computation, we send each set of variables $Y^{\varphi_{i}}$ to $\left\{y_{1}^{b_{i}}, y_{2}^{b_{i}}, \ldots\right\}$. Using plethysm defined as equation (2.3), we give the following definition.

Definition 5. Define a homomorphism $\rho: \mathbb{C}-\operatorname{span}\left\{S_{\boldsymbol{\lambda}} \mid \boldsymbol{\lambda} \in \mathcal{P}^{\Theta}\right\} \rightarrow \Lambda_{\mathbb{C}}(Y)$ as follows:

$$
\rho\left(h_{a}\left[Y^{\varphi}\right]\right)=h_{a}\left[p_{b}[Y]\right], \forall \varphi \in \Theta, b=\operatorname{deg}(\varphi) \text {. }
$$


The image of the homomorphism doesn't differentiate the sets of variables and therefore simplifies the computation. Applying the homomorphism does lose information on the characteristics of characters in $G_{n}$. However, as we will see later on in Theorem 17 and Corollary 18, if we consider the induced characters from $U_{n}$ to $G_{n}$, then the homomorphism carries all the information and this will be discussed later in this section.

Since $\operatorname{ch}\left(\chi_{\left(q_{1}, \ldots, q_{n-1}\right)}^{(n)} \uparrow_{U_{n}}^{G_{n}}\right)$ is independent of $q_{1}, \ldots, q_{n-1}$, and only depends on $n$ for $n \geqslant 1$, we can simply denote $\rho\left(\operatorname{ch}\left(\chi_{\left(q_{1}, \ldots, q_{n-1}\right)}^{(n)} \uparrow_{U_{n}}^{G_{n}}\right)\right)$ by $\rho_{n}$ and set $\rho_{0}=1$. We also use $\rho_{\left[m_{i, 1}, \ldots, m_{i, n}\right]}$ to denote the results of applying $\rho$ on the sum of all different products in the form of (3.4) for the same index list $\left[m_{i, 1}, \ldots, m_{i, n}\right]$, i.e.

$$
\rho_{\left[m_{i, 1}, \ldots, m_{i, n}\right]}:=\frac{l_{q}(i)\left(l_{q}(i)-1\right) \cdots\left(l_{q}(i)-m_{i}+1\right)}{m_{i, 1} ! m_{i, 2} ! \cdots m_{i, n} !}\left(h_{1}\left[p_{i}\right]\right)^{m_{i, 1}} \cdots\left(h_{n}\left[p_{i}\right]\right)^{m_{i, n}} .
$$

Applying $\rho$ to both sides of (3.2) we get

$$
\rho_{n}=\sum_{\sum_{i, j=1}^{n}} \rho_{m_{i, j} \times j \times i=n} \rho_{\left[m_{1,1}, \ldots, m_{1, n}\right]} \cdots \rho_{\left[m_{n, 1}, \ldots, m_{n, n}\right]} .
$$

The following theorem follows naturally.

Theorem 6. Let $C H_{q}(t)$ denote the generating function for $\rho_{n}$ :

$$
C H_{q}(t)=1+\rho_{1} t+\rho_{2} t^{2}+\cdots=\sum_{n \geqslant 0} \rho_{n} t^{n} .
$$

Then we have

$$
C H_{q}(t)=\prod_{i \geqslant 1}\left(\prod_{j \geqslant 1}\left(1-y_{j}^{i} t^{i}\right)^{-1}\right)^{l_{q}(i)}=\prod_{i \geqslant 1} \prod_{j \geqslant 1}\left(1-y_{j}^{i} t^{i}\right)^{-l_{q}(i)} .
$$

Proof. Since for every $i \geqslant 1$,

$$
\begin{aligned}
\prod_{j \geqslant 1}\left(1-y_{j}^{i} t^{i}\right)^{-1} & =\sum_{a \geqslant 0} h_{a}\left[y_{1}^{i}, y_{2}^{i}, \ldots\right] t^{a \cdot i} \\
& =1+\left(h_{1}\left[p_{i}\right]\right) \cdot t^{i}+\left(h_{2}\left[p_{i}\right]\right) \cdot t^{2 i}+\cdots .
\end{aligned}
$$

We have

$$
\begin{aligned}
& \left(\prod_{j \geqslant 1}\left(1-y_{j}^{i} t^{i}\right)^{-1}\right)^{l_{q}(i)} \\
& =\left(1+\left(h_{1}\left[p_{i}\right]\right) t^{i}+\left(h_{2}\left[p_{i}\right]\right) t^{2 i}+\cdots\right)^{l_{q}(i)} \\
& =\sum_{\substack{m_{i, 1}+m_{i, 2}+\cdots+m_{i, n}=m_{i} \\
0 \leqslant m_{i} \leqslant l_{q}(i)}}\left(\begin{array}{c}
l_{q}(i) \\
m_{i}
\end{array}\right)\left(\begin{array}{c}
m_{i} \\
m_{i, 1} m_{i, 2} \cdots m_{i, n}
\end{array}\right) \\
& \quad \\
& \quad \sum_{\substack{m_{i, 1}+m_{i, 2}+\cdots+m_{i, n}=m_{i} \\
0 \leqslant m_{i} \leqslant l_{q}(i)}} \rho_{\left[m_{i, 1}, \ldots, m_{i, n}\right]} \cdot t^{\left(m_{i, 1}+2 m_{i, 2}+\cdots+n \cdot m_{i, n}\right) \cdot i} \cdot
\end{aligned}
$$


From (3.5) we see that the coefficient of $t^{n}$ in the product $\prod_{i \geqslant 1}\left(\prod_{j \geqslant 1}\left(1-y_{j}^{i} t^{i}\right)^{-1}\right)^{l_{q}(i)}$ is exactly equal to $\rho_{n}$ for $n \geqslant 1$. Thus we get the Theorem.

\section{Theorem 7.}

$$
\prod_{i \geqslant 1} \prod_{j \geqslant 1}\left(1-y_{j}^{i} t^{i}\right)^{-l_{q}(i)}=\frac{\prod_{j \geqslant 1}\left(1-y_{j} t\right)}{\prod_{j \geqslant 1}\left(1-y_{j} q t\right)} .
$$

Proof. From equation (3.3) we know that when $i=1$,

$$
\prod_{j \geqslant 1}\left(1-y_{j} t\right)^{-l_{q}(1)} \prod_{j \geqslant 1}\left(1-y_{j} t\right)^{-1}=\prod_{j \geqslant 1}\left(1-y_{j} t\right)^{-L_{q}(1)}
$$

and when $i \geqslant 2$,

$$
\prod_{j \geqslant 1}\left(1-y_{j}^{i} t^{i}\right)^{-l_{q}(i)}=\prod_{j \geqslant 1}\left(1-y_{j} t\right)^{-L_{q}(i)} .
$$

Thus the above identity is equivalent to the identity

$$
\prod_{i \geqslant 1} \prod_{j \geqslant 1}\left(1-y_{j}^{i} t^{i}\right)^{L_{q}(i)}=\prod_{j \geqslant 1}\left(1-y_{j} q t\right)
$$

where $L_{q}(i)$ denotes the number of irreducible polynomials over $\mathbb{F}_{q}$ for $i \geqslant 1$, as we stated before.

Equation (3.7) is a classical identity which could be found, e.g. in [8] page 171. Here we give a direct computational proof by taking the logarithm on both sides of (3.7) and showing they are equal.

$$
\begin{aligned}
\ln \left(\prod_{i \geqslant 1} \prod_{j \geqslant 1}\left(1-y_{j}^{i} t^{i}\right)^{L_{q}(i)}\right) & =\sum_{j \geqslant 1}\left(\sum_{i \geqslant 1} L_{q}(i) \ln \left(1-y_{j}^{i} t^{i}\right)\right) \\
& =\sum_{j \geqslant 1}\left(\sum_{i \geqslant 1} L_{q}(i)\left(\sum_{r \geqslant 1} \frac{\left(y_{j}^{i} t^{i}\right)^{r}}{r}\right)\right) \\
& =\sum_{j \geqslant 1}\left(\sum_{i \geqslant 1} \sum_{r \geqslant 1} L_{q}\left(\frac{i \cdot r}{r}\right) \cdot \frac{i \cdot r}{r} \cdot \frac{y_{j}^{(i \cdot r)} \cdot t^{(i \cdot r)}}{i \cdot r}\right) \\
& =\sum_{j \geqslant 1}\left(\sum_{\substack{N \geqslant 1 \\
N=i \cdot r}} \frac{y_{j}^{N} \cdot t^{N}}{N}\left(\sum_{r \mid N} L_{q}\left(\frac{N}{r}\right) \cdot \frac{N}{r}\right)\right) \\
& =\sum_{j \geqslant 1}\left(\sum_{\substack{N \geqslant 1 \\
N=i \cdot r}} \frac{y_{j}^{N} \cdot t^{N}}{N} \cdot q^{N}\right) \\
& =\sum_{j \geqslant 1}\left(\ln \left(1-y_{j} q t\right)=\ln \left(\prod_{j \geqslant 1}\left(1-y_{j} q t\right)\right) .\right.
\end{aligned}
$$

Thus we get (3.7). 
Theorem (6) and Theorem (7) together yield the formula for the generating function of $\rho_{n}$ as follows:

$$
C H_{q}(t)=\prod_{j \geqslant 1} \frac{1-y_{j} t}{1-y_{j} q t} .
$$

Before we link it to Hall-Littlewood polynomials, we give a recurrence relation for $\rho_{n}$ using formula (3.8).

Corollary 8. For every $n \geqslant 1$, we have

$$
\rho_{n}=\left(q^{n}-1\right) h_{n}-\rho_{n-1} h_{1}-\rho_{n-2} h_{2}-\cdots-\rho_{1} h_{n-1} .
$$

Proof. From (3.8) we have

$$
C H_{q}(t) \times \Omega[t Y]=\prod_{j \geqslant 1}\left(1-y_{j} q t\right)^{-1} .
$$

Comparing the coefficients of $t^{n}$ on both sides, we get

$$
\rho_{0} h_{n}+\rho_{1} h_{n-1}+\cdots+\rho_{n} h_{0}=q^{n} h_{n},
$$

which yields the theorem.

\section{Example 9.}

$$
\begin{aligned}
\rho_{1} & =(q-1) h_{1} ; \\
\rho_{2} & =\left(q^{2}-1\right) h_{2}-\rho_{1} h_{1} \\
& =\left(q^{2}-1\right) h_{2}-(q-1) h_{1,1} \\
& =(q-1)\left[(q+1) h_{2}-h_{1,1}\right] ; \\
\rho_{3} & =\left(q^{3}-1\right) h_{3}-\rho_{1} h_{2}-\rho_{2} h_{1} \\
& =\left(q^{3}-1\right) h_{3}-(q-1) h_{2,1}-\left(q^{2}-1\right) h_{2,1}+(q-1) h_{1,1,1} \\
& =(q-1)\left[\left(q^{2}+q+1\right) h_{3}-(q+2) h_{2,1}+h_{1,1,1}\right] .
\end{aligned}
$$

From the above examples we notice that the coefficients of $h_{\lambda}$ are in $\pm \mathbb{N}[q] \times(q-1)$. Let $\left[h_{\lambda}\right] \rho_{n}$ denote the coefficients of $h_{\lambda}$ in the expansion of $\rho_{n}$. In particular, we have $\left[h_{n}\right] \rho_{n}=q^{n}-1$ for all $n \geqslant 1$. The following corollary gives the recurrence relation on the coefficients.

Corollary 10. For any $\lambda=\left(a_{1}^{l_{1}}, a_{2}^{l_{2}}, \ldots, a_{k}^{l_{k}}\right) \vdash n$ with $l_{i} \geqslant 1$ for all $1 \leqslant i \leqslant k$ and $l(\lambda) \geqslant 2$, we have

$$
\left[h_{\lambda}\right] \rho_{n}=-\left[h_{\left(a_{1}^{l_{1}-1}, a_{2}^{l_{2}}, \ldots, a_{k}^{l_{k}}\right)}\right] \rho_{n-a_{1}}-\left[h_{\left(a_{1}^{l_{1}}, a_{2}^{l_{2}-1}, \ldots, a_{k}^{l_{k}}\right)}\right] \rho_{n-a_{2}}-\cdots-\left[h_{\left(a_{1}^{l_{1}}, a_{2}^{l_{2}}, \ldots, a_{k}^{l_{k}-1}\right)}\right] \rho_{n-a_{k}}
$$

Here, if $l_{i}=1$ for some $1 \leqslant i \leqslant k$, then we set

$$
\left(a_{1}^{l_{1}}, \ldots, a_{i}^{l_{i}-1}, \ldots, a_{k}^{l_{k}}\right):=\left(a_{1}^{l_{1}}, \ldots, \hat{a}_{i}, \ldots, a_{k}^{l_{k}}\right),
$$

where $\hat{a}_{i}$ means simply remove $a_{i}$ from the partition $\lambda$. In particular, $\left[h_{\lambda}\right] \rho_{n} \in \pm \mathbb{N}[q] \times$ $(q-1)$, while the sign is given by $(-1)^{l(\lambda)-1}$. 
Proof. Equation (3.10) follows directly from Corollary 8 by comparing the coefficients of $h_{\lambda}$ from two sides. The claim that $\left[h_{\lambda}\right] \rho_{n}$ is in $\pm \mathbb{N}[q] \times(q-1)$ together with the sign property can be proved easily by using induction on equation (3.10).

Remark 11. Corollary 8 and Corollary 10 give an easy way of computing $\rho_{n}$ for every $n \geqslant 1$ simply by knowing $\left[h_{i}\right] \rho_{i}=q^{i}-1$ for every $i \geqslant 1$.

\section{Example 12.}

$$
\begin{aligned}
{\left[h_{2,1}\right] \rho_{3} } & =-\left[h_{1}\right] \rho_{1}-\left[h_{2}\right] \rho_{2} \\
& =-(q-1)-\left(q^{2}-1\right) \\
& =-(q-1)(q+2) . \\
{\left[h_{1,1,1}\right] \rho_{3} } & =-\left[h_{1,1}\right] \rho_{2}=\left[h_{1}\right] \rho_{1} \\
& =q-1 .
\end{aligned}
$$

Now back to our formula (3.8). We rewrite it in the following form so that we can easily use the generating function for $\boldsymbol{q}_{r}$ as in equation (2.2).

$$
\begin{aligned}
C H_{q}(t) & =\prod_{j \geqslant 1} \frac{1-y_{j} t}{1-y_{j} q t}=\prod_{j \geqslant 1} \frac{1-y_{j} \cdot \frac{1}{q} \cdot(q t)}{1-y_{j} \cdot(q t)} \\
& =\sum_{r \geqslant 0} \boldsymbol{q}_{r}\left[Y ; q^{-1}\right] q^{r} t^{r}
\end{aligned}
$$

where $Y=\left\{y_{1}, y_{2}, \ldots\right\}$. Comparing the coefficients from two sides we get the following corollary.

\section{Corollary 13.}

$$
\begin{aligned}
\rho_{n} & =\boldsymbol{q}_{n}\left[Y ; q^{-1}\right] q^{n}=\left(1-q^{-1}\right) P_{n}\left[Y ; q^{-1}\right] q^{n} \\
& =q^{n-1}(q-1) P_{n}\left[Y ; q^{-1}\right]=q^{n-1}(q-1) \tilde{P}_{n}[Y ; q] .
\end{aligned}
$$

Corollary 13 gives the connection between the image of the homomorphism $\rho$ on the characteristic map of $\chi_{\left(q_{1}, \ldots, q_{n-1}\right)}^{(n)} \uparrow_{U_{n}}^{G_{n}}$ and the Hall-Littlewood symmetric functions. As we mentioned in the introduction, we refer to this result as a plethysm formula on $\operatorname{ch}\left(\chi_{\left(q_{1}, \ldots, q_{n-1}\right)}^{(n)} \uparrow_{U_{n}}^{G_{n}}\right)$.

For any linear supercharacter $[10,9,1]$ of $U_{n}$, there is a unique way to decompose the indexed set partition into connected components. We denote a linear supercharacter with $k$ connected components by $\chi_{\overrightarrow{q_{1}}, \ldots, \overrightarrow{q_{k}}}^{n_{1}\left|n_{2}\right| \ldots \mid n_{k}}$, where for $i$ from 1 to $k$, each $n_{i}$ counts the size of the $i^{t h}$ connected component, and $\vec{q}_{i}=\left(q_{i, 1}, \ldots, q_{i, n_{i}-1}\right) \in\left(\mathbb{F}_{q}^{\times}\right)^{n_{i}-1}$ denotes the labels of the arcs for the $i^{\text {th }}$ connected component. The following corollary follows from the property of the linear supercharacters $[10,9,1]$. 


\section{Corollary 14.}

$$
\rho\left(\operatorname{ch}\left(\chi_{\overrightarrow{q_{1}, \ldots, q_{k}}}^{n_{1}\left|n_{2}\right| \ldots \mid n_{k}} \uparrow_{U_{n}}^{G_{n}}\right)\right)=\prod_{i=1}^{k} \rho_{n_{i}} .
$$

Example 15. For the following linear supercharacter of $U_{6}$

$$
\chi_{\overrightarrow{q_{1}}, \overrightarrow{q_{2}}, \overrightarrow{q_{3}}}^{1|2| 3}=\chi^{i} \stackrel{\overbrace{2}^{q_{3}, 1}}{\overbrace{3}} \overbrace{\underbrace{}_{5}}^{q_{3,1} q_{3,2}}
$$

where $\overrightarrow{q_{1}}=(), \overrightarrow{q_{2}}=\left(q_{2,1}\right), \overrightarrow{q_{3}}=\left(q_{3,1}, q_{3,2}\right)$ and $q_{2,1}, q_{3,1}, q_{3,2} \in \mathbb{F}_{q}^{\times}$, we have

$$
\rho \circ \operatorname{ch}\left(\chi_{\overrightarrow{q_{1}}, \overrightarrow{q_{2}}, \overrightarrow{q_{3}}}^{1|2| 3} \uparrow_{U_{6}}^{G_{6}}\right)=\rho_{1} \rho_{2} \rho_{3} .
$$

Let the transition matrix between $\left\{m_{\lambda}[X]\right\}_{\lambda \vdash n}$ and $\left\{p_{\mu}[X]\right\}_{\mu \vdash n}$ be $C_{\lambda, \mu}$, i.e.

$$
m_{\lambda}[X]=\sum_{\mu} C_{\lambda, \mu} p_{\mu}[X]
$$

Define $m_{\lambda}[q-1]$ by the following equation

$$
m_{\lambda}[q-1]=\sum_{\mu} C_{\lambda, \mu} p_{\mu}[q-1]
$$

where $p_{n}[q-1]=q^{n}-1$ for every $n \geqslant 1$, and $p_{\mu}[q-1]=p_{\mu_{1}}[q-1] \cdots p_{\mu_{l}}[q-1]$ for $\mu=\left(\mu_{1}, \ldots, \mu_{l}\right)$.

Remark 16. Using the orthogonal relation between the bases $\left\{m_{\lambda}\right\}$ and $\left\{h_{\mu}\right\}$, we give another expression for $\rho_{n}$ as follows:

$$
\rho_{n}=\sum_{\lambda \vdash n} m_{\lambda}[q-1] \times h_{\lambda}[Y]
$$

Proof. Using the notation in Section 2.1, we have

$$
\begin{aligned}
\Omega[q t Y]= & \prod_{j \geqslant 1} \frac{1}{1-y_{j} q t}, \quad \Omega[-t Y]=\prod_{j \geqslant 1}\left(1-y_{j} t\right) . \\
C H_{q}(t) & =\prod_{j \geqslant 1} \frac{1-y_{j} t}{1-y_{j} q t} \\
& =\Omega[(q-1) t Y] \\
& =\sum_{n \geqslant 0}\left(\sum_{\lambda \vdash n} m_{\lambda}[q-1] \cdot h_{\lambda}[Y]\right) t^{n} .
\end{aligned}
$$


As we mentioned above, it seems that we lose information by applying $\rho$ to the characteristic map of $\chi_{\left(q_{1}, \ldots, q_{n-1}\right)}^{(n)} \uparrow_{U_{n}}^{G_{n}}$. However, since $\chi_{\left(q_{1}, \ldots, q_{n-1}\right)}^{(n)} \uparrow_{U_{n}}^{G_{n}}$ is a character induced from $U_{n}$, this allows us to express $\operatorname{ch}\left(\chi_{\left(q_{1}, \ldots, q_{n-1}\right)}^{(n)} \uparrow_{U_{n}}^{G_{n}}\right)$ in basis $\left\{\tilde{P}_{\boldsymbol{\mu}} \mid \boldsymbol{\mu} \in \mathcal{P}^{\Phi}\right\}$ from the result of applying $\rho$. To show this fact, we first introduce the following homomorphism on symmetric function ring defined in [7]:

$$
\omega: \Lambda_{\mathbb{C}}(Y) \rightarrow \Lambda_{\mathbb{C}}(Y)
$$

by

$$
\omega\left(e_{r}[Y]\right)=h_{r}[Y], \text { for all } r \geqslant 0 .
$$

The map $\omega$ is a well known involution and automorphism on $\Lambda_{\mathbb{C}}(Y)$. Also, we have

$$
\omega\left(p_{r}[Y]\right)=(-1)^{r-1} p_{r}[Y], \text { for all } r \geqslant 0 .
$$

The following theorem illustrates the relation between applying the homomorphism $\rho$ to the characteristic map in basis $\left\{S_{\boldsymbol{\lambda}} \mid \boldsymbol{\lambda} \in \mathcal{P}^{\Theta}\right\}$ and the projection on the characteristic map in basis $\left\{\tilde{P}_{\boldsymbol{\mu}} \mid \boldsymbol{\mu} \in \mathcal{P}^{\Phi}\right\}$ to the space $\Lambda_{\mathbb{C}}\left(X_{x-1}\right)$.

Theorem 17. The following diagram commutes:

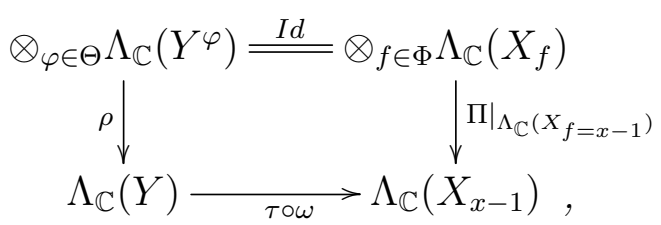

where $\tau$ is the map of changing variables $y_{i}$ into $X_{i, x-1}$ for $i=1,2, \ldots$, and $\left.\Pi\right|_{\Lambda_{\mathbb{C}}\left(X_{f=x-1}\right)}$ is the projection to the space $\Lambda_{\mathbb{C}}\left(X_{x-1}\right)$.

Proof. Recall equation (2.4)

$$
p_{k}\left[Y^{\varphi}\right]=(-1)^{n-1} \sum_{x \in M_{n}} \xi(x) p_{n / d\left(f_{x}\right)}\left[X_{f_{x}}\right],
$$

where $\xi \in \varphi, x \in f_{x}$ and $n=k \times d(\varphi)$. If we apply $\rho$ to $p_{k}\left[Y^{\varphi}\right]$ we get $p_{n}[Y]$. Applying the projection map $\left.\Pi\right|_{\Lambda_{\mathbb{C}}\left(X_{f=x-1}\right)}$ on the right-hand side of equation (2.4) yields $(-1)^{n-1} p_{n}\left[X_{x-1}\right]$. Since $\left\{p_{n}: n=1,2, \ldots\right\}$ are algebraically independent over $\mathbb{C}$ and $\left\{p_{\lambda}: \lambda\right.$ a partition $\}$ form a basis of the symmetric function ring, we get the theorem.

Since $\Lambda_{\mathbb{C}}$ has two bases $\left\{S_{\boldsymbol{\lambda}} \mid \boldsymbol{\lambda} \in \mathcal{P}^{\Theta}\right\}$ and $\left\{\tilde{P}_{\boldsymbol{\mu}} \mid \boldsymbol{\mu} \in \mathcal{P}^{\Phi}\right\}$, we use $\operatorname{ch}\left(\chi \uparrow_{U_{n}}^{G_{n}}\right)\left(X_{f}: f \in \Phi\right)$ to denote the expression in terms of basis $\left\{\tilde{P}_{\boldsymbol{\mu}} \mid \boldsymbol{\mu} \in \mathcal{P}^{\Phi}\right\}$, and $\operatorname{ch}\left(\chi \uparrow_{U_{n}}^{G_{n}}\right)\left(Y^{\varphi}: \varphi \in \Theta\right)$ the expression in terms of basis $\left\{S_{\boldsymbol{\lambda}} \mid \boldsymbol{\lambda} \in \mathcal{P}^{\Theta}\right\}$. We then have the following identity.

\section{Corollary 18.}

$$
\begin{aligned}
\operatorname{ch}\left(\chi_{\left(q_{1}, \ldots, q_{n-1}\right)}^{(n)} \uparrow_{U_{n}}^{G_{n}}\right)\left(X_{f}: f \in \Phi\right) & =\tau \circ \omega\left(\rho_{n}\right) \\
& =q^{n-1}(q-1) \omega\left(\tilde{P}_{n}\left[X_{x-1} ; q\right]\right) .
\end{aligned}
$$


Proof. From the definition of the induced character by equation (2.5) we know that

$$
\chi_{\left(q_{1}, \ldots, q_{n-1}\right)}^{(n)} \uparrow_{U_{n}}^{G_{n}}(g)=0
$$

for all $g \in G_{n}$ which are not similar to any unipotent upper-triangular matrices. Notice that the characteristic polynomial for all matrices in $U_{n}$ is $(x-1)^{n}$. Since similar matrices have the same characteristic polynomial, $\chi_{\left(q_{1}, \ldots, q_{n-1}\right)}^{(n)} \uparrow_{U_{n}}^{G_{n}}$ could possibly take nonzero values only on those matrices in $G_{n}$ with characteristic polynomials equal to $(x-1)^{n}$. We then have

$$
\operatorname{ch}\left(\chi_{\left(q_{1}, \ldots, q_{n-1}\right)}^{(n)} \uparrow_{U_{n}}^{G_{n}}\right)\left(X_{f}: f \in \Phi\right) \in \Lambda_{\mathbb{C}}\left(X_{x-1}\right),
$$

and so

$$
\left.\Pi\right|_{\Lambda_{\mathbb{C}}\left(X_{f=x-1}\right)}\left[\operatorname{ch}\left(\chi_{\left(q_{1}, \ldots, q_{n-1}\right)}^{(n)} \uparrow_{U_{n}}^{G_{n}}\right)\right]=\operatorname{ch}\left(\chi_{\left(q_{1}, \ldots, q_{n-1}\right)}^{(n)} \uparrow_{U_{n}}^{G_{n}}\right) .
$$

By theorem 17 we obtain the corollary.

Remark 19. From the proof of Corollary 18 we conclude that for any character $\chi$ of $U_{n}$, if we induce $\chi$ from $U_{n}$ to $G_{n}$, then we have

$$
\operatorname{ch}\left(\chi \uparrow \uparrow_{U_{n}}^{G_{n}}\right)\left(X_{f}: f \in \Phi\right)=\tau \circ \omega \circ \rho\left(\operatorname{ch}\left(\chi \uparrow_{U_{n}}^{G_{n}}\right)\left(Y^{\varphi}: \varphi \in \Theta\right)\right) .
$$

For $\lambda=\left(\lambda_{1}, \ldots, \lambda_{l}\right)$ let $\rho_{\lambda}=\rho_{\lambda_{1}} \rho_{\lambda_{2}} \ldots \rho_{\lambda_{l}}$. By Corollary 13, since $\rho_{n}=q^{n-1}(q-$ 1) $P_{n}\left[Y ; q^{-1}\right]$, we know that $\left\{\rho_{\lambda}\right\}$ forms a basis for the symmetric function $\operatorname{ring} \Lambda_{\mathbb{C}}(Y)$. Thus $\rho\left(\operatorname{ch}\left(\chi \uparrow_{U_{n}}^{G_{n}}\right)\right)$ can be written as $\rho\left(\operatorname{ch}\left(\chi \uparrow_{U_{n}}^{G_{n}}\right)\right)=\sum_{\lambda \vdash n} C_{\lambda} \rho_{\lambda}$, where $C_{\lambda} \in \mathbb{C}$. We then define a map as follows.

Definition 20. Define $\hat{\rho}: \Lambda_{\mathbb{C}}(Y) \rightarrow \mathbb{C}$-span $\left\{S_{\boldsymbol{\lambda}} \mid \boldsymbol{\lambda} \in \mathcal{P}^{\Theta}\right\}$ by

$$
\begin{aligned}
\hat{\rho}\left(\rho_{n}\right) & :=\sum_{\substack{\boldsymbol{\lambda} \in \mathcal{P}^{\Theta} \\
h t(\boldsymbol{\lambda})=1}} S_{\boldsymbol{\lambda}} \\
& =\operatorname{ch}\left(\chi_{\left(q_{1}, \ldots, q_{n-1}\right)}^{(n)} \uparrow_{U_{n}}^{G_{n}}\right),
\end{aligned}
$$

and

$$
\hat{\rho}\left(\rho_{\lambda}\right)=\hat{\rho}\left(\rho_{\lambda_{1}}\right) \hat{\rho}\left(\rho_{\lambda_{2}}\right) \ldots \hat{\rho}\left(\rho_{\lambda_{l}}\right),
$$

where $\lambda=\left(\lambda_{1}, \ldots, \lambda_{l}\right)$.

Proposition 21. For a fixed finite field $\mathbb{F}_{q}$ and a character $\chi$ of $U_{n}$, we have

$$
(\hat{\rho} \circ \rho)\left(\operatorname{ch}\left(\chi \uparrow_{U_{n}}^{G_{n}}\right)\right)=\operatorname{ch}\left(\chi \uparrow \uparrow_{U_{n}}^{G_{n}}\right) .
$$

Proof. First of all, we have $\hat{\rho} \circ \rho\left(\operatorname{ch}\left(\chi_{\left(q_{1}, \ldots, q_{n-1}\right)}^{(n)} \uparrow_{U_{n}}^{G_{n}}\right)\right)=\hat{\rho}\left(\rho_{n}\right)=\operatorname{ch}\left(\chi_{\left(q_{1}, \ldots, q_{n-1}\right)}^{(n)} \uparrow_{U_{n}}^{G_{n}}\right)$. From Theorem 17 and Remark 19 we know that $\rho$ restricted on the characteristics of induced characters from $U_{n}$ to $G_{n}$ is an isomorphism. Since $\left\{\rho_{n}\right\}_{n \in \mathbb{N}}$ forms an algebraic basis and for all $\operatorname{ch}\left(\chi \uparrow_{U_{n}}^{G_{n}}\right), \rho$ must have a unique inverse, we get the proposition. 
Suppose $\rho\left(\operatorname{ch}\left(\chi \uparrow_{U_{n}}^{G_{n}}\right)\right)=\sum_{\lambda \vdash n} C_{\lambda} \rho_{\lambda}$, where $C_{\lambda} \in \mathbb{C}$. From the definition of $\hat{\rho}$ we get

$$
\begin{aligned}
\hat{\rho} \circ \rho\left(\operatorname{ch}\left(\chi \uparrow_{U_{n}}^{G_{n}}\right)\right) & =\sum_{\lambda \vdash n} C_{\lambda}\left(\hat{\rho}\left(\rho_{\lambda}\right)\right) \\
& =\sum_{\lambda \vdash n} C_{\lambda} \hat{\rho}\left(\rho_{\lambda_{1}}\right) \hat{\rho}\left(\rho_{\lambda_{2}}\right) \ldots \hat{\rho}\left(\rho_{\lambda_{l}}\right) .
\end{aligned}
$$

Using Proposition 21 we get the following corollary.

Corollary 22. For a fixed finite field $\mathbb{F}_{q}$ and a character $\chi$ of $U_{n}$, suppose $\operatorname{ch}\left(\chi \uparrow_{U_{n}}^{G_{n}}\right)=$ $\sum_{\lambda \vdash n} C_{\lambda} \rho_{\lambda}$ where $C_{\lambda} \in \mathbb{C}$. We have

$$
\begin{aligned}
\operatorname{ch}\left(\chi \uparrow_{U_{n}}^{G_{n}}\right) & =\sum_{\lambda \vdash n} C_{\lambda} \rho_{\lambda} \\
& =\sum_{\lambda \vdash n} C_{\lambda}\left(\sum_{\substack{\boldsymbol{\lambda}^{(1)} \in \mathcal{P}_{\lambda_{1}}^{\Theta} \\
h t\left(\boldsymbol{\lambda}^{(1)}\right)=1}} S_{\boldsymbol{\lambda}^{(1)}}\right)\left(\sum_{\substack{\boldsymbol{\lambda}^{(2)} \in \mathcal{P}_{\lambda_{2}}^{\Theta} \\
h t\left(\boldsymbol{\lambda}^{(2)}\right)=1}} S_{\boldsymbol{\lambda}^{(2)}}\right) \cdots\left(\sum_{\substack{\boldsymbol{\lambda}^{(l)} \in \mathcal{P}_{\lambda_{l}}^{\Theta} \\
h t\left(\boldsymbol{\lambda}^{(l)}\right)=1}} S_{\boldsymbol{\lambda}^{(l)}}\right) .
\end{aligned}
$$

Remark 23. It is difficult to get an expression for $\operatorname{ch}\left(\chi \uparrow_{U_{n}}^{G_{n}}\right)$ in terms of basis $\left\{S_{\boldsymbol{\lambda}} \mid \boldsymbol{\lambda} \in \mathcal{P}^{\Theta}\right\}$, which gives the irreducible decomposition of the induced character. However, if we know the image of $\rho$ on the characteristic map of $\chi \uparrow_{U_{n}}^{G_{n}}$, we may use $\hat{\rho}$ to get the irreducible decomposition of $\operatorname{ch}\left(\chi \uparrow_{U_{n}}^{G_{n}}\right)$. We hope these results can contribute to research in this problem. We list some open problems in Section 4.

\section{Further Questions}

The induced characters that we are studying in this paper are very special. A natural question to ask is: "can we give a nice formula for the characteristics of all the induced supercharacters from $U_{n}$ to $G_{n}$ ?" Zelevinsky [14] and Thiem and Vinroot [11] have worked on the case of degenerate Gelfand-Graev characters. The problem of how the generalized Gelfand-Graev representations of the finite unitary group decompose is still open.

The generalized Gelfand-Graev representations, which are defined by Kawanaka [6], are obtained by inducing certain irreducible representations from a unipotent subgroup [11]. Here the supercharacters that we are considering are more general than the case of the generalized Gelfand-Graev representations. We hope that the ideas and results developed in this paper could help to work on this problem.

Below we give some examples of computations of $\rho$ acting on the characteristic map of induced supercharacters.

Example 24. For $q=2$, we have

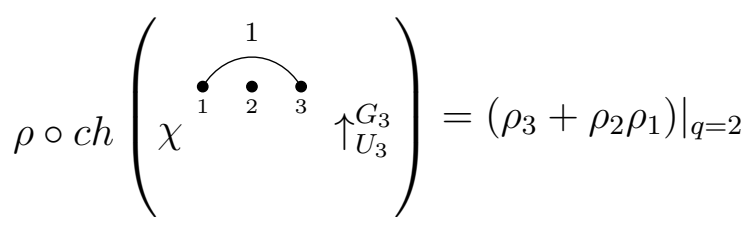

THE ELECTRONiC JOURNAL Of COMBINATORics 20(3) (2013), \#P12 


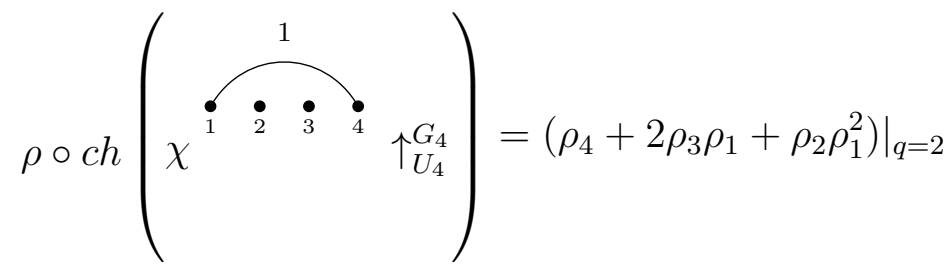

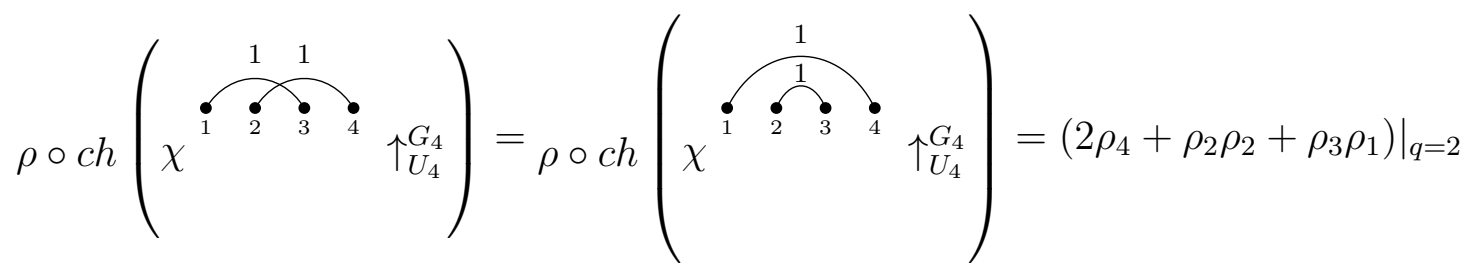

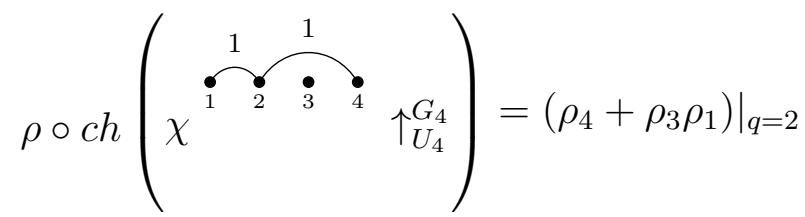

Inspired by these results, we propose the following conjecture and state some questions that remain open.

Conjecture 25. For a fixed finite field $\mathbb{F}_{q}$ and a supercharacter $\chi$ of $U_{n}$, we have

$$
\rho\left(\operatorname{ch}\left(\chi \uparrow \uparrow_{U_{n}}^{G_{n}}\right)\right) \in \mathbb{N}\left[\rho_{1}, \ldots, \rho_{n}\right] .
$$

The conjecture is interesting in the sense that not every character of a larger group can be represented as a non-negative integer combination of characters induced from linear characters of subgroups. It is interesting to know which kind of characters have that property. If the above conjecture is true, then the following remark is meaningful.

Remark 26. For a fixed finite field $\mathbb{F}_{q}$ and a character $\chi$ of $U_{n}$, suppose $\rho\left(\operatorname{ch}\left(\chi \uparrow_{U_{n}}^{G_{n}}\right)\right)=$ $\sum_{\lambda \vdash n} C_{\lambda} \rho_{\lambda}$ where $C_{\lambda} \in \mathbb{C}$. We have

$$
\operatorname{dim}(\chi)=\sum_{\lambda \vdash n} C_{\lambda}
$$

Proof. From Corollary 14 we have

$$
\chi \uparrow_{U_{n}}^{G_{n}}=\sum_{\lambda \vdash n} C_{\lambda}\left(\chi_{\overrightarrow{q_{1}}, \ldots, \vec{q}_{l}}^{\lambda_{1}\left|\lambda_{2}\right| \ldots \mid \lambda_{l}} \uparrow_{U_{n}}^{G_{n}}\right)=\left(\sum_{\lambda \vdash n} C_{\lambda} \chi_{\overrightarrow{q_{1}}, \ldots, \bar{q}_{l}}^{\lambda_{1}\left|\lambda_{2}\right| \ldots \mid \lambda_{l}}\right) \uparrow_{U_{n}}^{G_{n}},
$$

where $\vec{q}_{i}=\left(q_{i, 1}, \ldots, q_{i, \lambda_{i}-1}\right) \in\left(\mathbb{F}_{q}^{\times}\right)^{\lambda_{i}-1}$. So we have

$$
\operatorname{dim}(\chi)=\operatorname{dim}\left(\sum_{\lambda \vdash n} C_{\lambda} \chi_{\overrightarrow{q_{1}}, \ldots, \overrightarrow{q_{l}}}^{\lambda_{1}\left|\lambda_{2}\right| \ldots \mid \lambda_{l}}\right)=\sum_{\lambda \vdash n} C_{\lambda} \operatorname{dim}\left(\chi_{\overrightarrow{q_{1}}, \ldots, \vec{q}_{l}}^{\lambda_{1}\left|\lambda_{2}\right| \ldots \mid \lambda_{l}}\right) .
$$

Since $\operatorname{dim}\left(\chi_{\overrightarrow{q_{1}}, \ldots, \overrightarrow{q_{l}}}^{\lambda_{1}\left|\lambda_{2}\right| \ldots \mid \lambda_{l}}\right)=1$, equation (4.1) follows. 
Question 27. For a fixed finite field $\mathbb{F}_{q}$ and a supercharacter $\chi$ of $U_{n}$, find a formula for the image of the homomorphism $\rho$ on the characteristic map of $\chi \uparrow_{U_{n}}^{G_{n}}$,

$$
\rho \circ \operatorname{ch}\left(\chi \uparrow_{U_{n}}^{G_{n}}\right)=\sum_{\lambda \vdash n} C_{\lambda} \rho_{\lambda}
$$

where $\rho_{\lambda}=\rho_{\lambda_{1}} \rho_{\lambda_{1}} \ldots \rho_{\lambda_{l}}$ for $\lambda=\lambda_{1}, \ldots, \lambda_{l}$. It would be nice to give a combinatorial formula for the coefficient $C_{\lambda}$, since the examples above suggest a possibility of some rules.

Remark 28. If we have the formula of $\rho\left(\operatorname{ch}\left(\chi \uparrow_{U_{n}}^{G_{n}}\right)\right)$, we can easily get the expression for the characteristic map of $\chi \uparrow_{U_{n}}^{G_{n}}$ in terms of basis $\left\{\tilde{P}_{\boldsymbol{\mu}} \mid \boldsymbol{\mu} \in \mathcal{P}^{\Phi}\right\}$ by Remark 19. We may also use $\hat{\rho}$ to get an expression in the basis $\left\{S_{\boldsymbol{\lambda}} \mid \boldsymbol{\lambda} \in \mathcal{P}^{\Theta}\right\}$ by Corollary 22 .

Question 29. Up to now the induced representations that we are considering are in characteristic zero. Another problem we can think about is what happens in characteristic $p$ case.

\section{Acknowledgements}

I am grateful to my advisor, Professor Nantel Bergeron, for his guidance and discussions on this research problem. I also want to thank the anonymous referee and Professor Mike Zabrocki for many useful suggestions.

\section{References}

[1] Marcelo Aguiar, Carlos Andre, Carolina Benedetti, Nantel Bergeron, Zhi Chen, Persi Diaconis, Anders Hendrickson, Samuel Hsiao, I. Martin Isaacs, Andrea Jedwab, Kenneth Johnson, Gizem Karaali, Aaron Lauve, Tung Le, Stephen Lewis, Huilan Li, Kay Magaard, Eric Marberg, Jean-Christophe Novelli, Amy Pang, Franco Saliola, Lenny Tevlin, Jean-Yves Thibon, Nathaniel Thiem, Vidya Venkateswaran, C. Ryan Vinroot, Ning Yan, Mike Zabrocki., Supercharacters, symmetric functions in noncommuting variables, and related Hopf algebras, Adv. Math. 229 (2012), no. 4, 2310-2337.

[2] C. AndrÉ, Basic characters of the unitriangular group, J. Algebra 175 (1995), $287-319$.

[3] A. M. Garsia And G. Tesler, Plethystic formulas for Macdonald q, t-Kostka coefficients, Adv. Math. 123 (1996), no. 2, 144-222.

[4] I. M. Gelfand And M. I. GRAev, Construction of irreducible representations of simple algebraic groups over a finite field, Dokl. Akad. Nauk SSSR 147 (1962), 529-532. 
[5] J. A. Green, The Characters of the finite general linear groups, Transactions of the American Mathematical Society, 80 (1955), 402-447.

[6] N. Kawanaka, Generalized Gelfand-Graev representations and Ennola duality, In Algebraic groups and related topics (Kyoto/Nagoya, 1983), 175-206, Adv. Stud. Pure Math., 6, North-Holland, Amsterdam, 1985.

[7] I.G. Macdonald, Symmetric Functions and Hall-Polynomials, Oxford Mathematical Monographs, Oxford Univ. Press, second edition (1995) 488p.

[8] Christophe Reutenauer, Free Lie Algebras, Oxford Science Publications, 2003.

[9] Nathaniel Thiem, Unipotent Hecke algebras: the structure, representation theory, and combinatorics, Ph.D Thesis, University of Wisconsin - Madison, 2004.

[10] Nathaniel Thiem, Branching rules in the ring of superclass functions of unipotent upper-triangular matrices, J. Algebraic Combin. 31 (2010), no. 2, 267-298.

[11] Nathaniel Thiem and C. Ryan Vinroot, Gelfand-Graev characters of the finite unitary groups, Electron. J. Combin. 16 (2009), no. 1, Research Paper 146, 37 pp.

[12] R. Steinberg, Lectures on Chevalley groups, mimeographed notes, Yale University, 1968.

[13] N. Yan, Representation theory of the finite unipotent linear groups, Unpublished Ph.D. Thesis, Department of Mathematics, University of Pennsylvania, 2001.

[14] A. V. Zelevinsky, Representations of finite classical groups. A Hopf algebra approach, Lec- ture Notes in Mathematics 869, Springer-Verlag, Berlin-New York, 1981. 